\title{
Enhanced COVID-19 data for improved prediction of survival
}

\author{
Wenhuan Zeng ${ }^{1 \dagger *}$, Anupam Gautam ${ }^{1,2 \dagger}$, Daniel H Huson ${ }^{1}$
}

1 Institute for Bioinformatics and Medical Informatics, University of Tübingen, Sand 14, Tübingen, 72076, Germany.

2 International Max Planck Research School 'From Molecules to Organisms', Tübingen, Germany.

$\dagger$ These authors contributed equally to this work.

* wenhuan.zeng@uni-tuebingen.de

\section{Abstract}

The current COVID-19 pandemic, caused by the rapid world-wide spread of the SARS-CoV-2 virus, is having severe consequences for human health and the world economy. The virus effects individuals quite differently, with many infected patients showing only mild symptoms, and others showing critical illness. To lessen the impact of the pandemic, one important question is which factors predict the death of a patient? Here, we construct an enhanced COVID-19 dataset by processing two existing databases (from Kaggle and WHO) and using natural language processing methods to enhance the data by adding local weather conditions and research sentiment.

\section{Author summary}

In this study, we contribute an enhanced COVID-19 dataset, which contains 183 samples and 43 features. Application of Extreme Gradient Boosting (XGBoost) on the enhanced dataset achieves $95 \%$ accuracy in predicting patients survival, with country-wise research sentiment, and then age and local weather, showing the most importance. All data and source code are available at

http://ab.inf .uni-tuebingen.de/publications/papers/COVID-19.

\section{Introduction}

The current COVID-19 pandemic, caused by the rapid world-wide spread of the SARS-CoV-2 virus, is affecting many aspects of society, in particular human health, but also social issues 1, 2], mental health and the economy 3]. Medical researchers, and researchers from different scientific fields, including immunology, genetics and bioinformatics, are studying the pandemic to find ways to slow its progression. Machine learning approaches are being utilized to understand aspects of the problem.

To date, most machine learning research on COVID-19 has used supervised learning methods or deep learning [4,5] to investigate which might be the important features to predict a predefined outcome. Running such approaches on the publicly available datasets is associated with difficulties that are due to the fact that features are collected depending on the needs of the data provider, which can be a source of bias. In particular, features that have high predictive value for the outcome for an infected 
patient, might be missing. Generally speaking, the presence or absence of features will impact the accuracy of a model.

The currently available COVID-19 data is missing features and we explore the effect of this by adding a number of features that might be important, so as to determine how this affects the accuracy of the model.

We used data on patients that tested positive for the virus and added new features based on (1) how different countries responded to the pandemic in terms of research sentiment (so as to calculate a weighted average polarity score for research abstracts per country) and (2) the local weather conditions when the patient was probably infected. We found that age is one of the most important factors when we have not incorporated these additional features based on the initial data.

However, after the addition of two new features, country-specific research sentiment, followed by local weather and age, came out to be the most important features. Recent publications suggest that the weather, as represented by the variables temperature and humidity, plays a role in COVID-19 6] and SARS [7].

To summarize, our main contributions are as follows:

- We demonstrate how to construct an enhanced set of COVID-19 features using additional available information.

- Using this enhanced dataset, we show that the Extreme Gradient Boosting (XGBoost) method achieves $95 \%$ accuracy in predicting a patient's survival.

- We show that country-specific research sentiment, followed by age and local weather and are the most important features.

\section{Materials}

We first compiled an initial dataset by combining data from two sources.

\section{Kaggle Novel Corona Virus 2019 dataset}

We used data from Kaggle available at its Novel Corona Virus 2019 Dataset portal (https://www.kaggle.com/sudalairajkumar/novel-corona-virus-2019-dataset), which contains day-level information on COVID-19 cases. The dataset includes features such as ID, age, sex, city, province and country. The dataset file

(COVID19_open_line_list.csv) was downloaded on 30/03/2020. All the rows that do not contain a value in the outcome column were dropped, resulting in 183 patient data rows out of 13,174 . The final dataset contains 183 patients from 16 countries. Further processing was carried out on this dataset (S1 File).

\section{WHO COVID-19 database}

We downloaded a database of literature on COVID-19 from the World Health Organization (WHO) web site (https://www.who.int/emergencies/diseases/novelcoronavirus-2019/global-research-on-novel-coronavirus-2019-ncov), on April 13, 2020. Of the 5,354 downloaded entries, we kept only those whose "Journal Name" and "DOI" fields were not blank, which resulted in 4,683 publications in 590 journals. We then analyzed these publications to determine the authors' institute and country (S2 File). 


\section{COVID-19 enhanced dataset}

In this paper, we present an enhanced COVID-19 dataset, which is based on the above described initial database. The data is enhanced by adding features that reflect the local weather and research sentiment in the country of the infected person, as described in the following (S3 File).

\section{Addition feature construction}

Database construction was performed as outlined in Fig 1. It has been demonstrated that there is a link between environmental factors and the development of COVID-19 [8]. Indeed, it seems reasonable to suspect that the weather conditions play a role.

Hence, we collected temperature, humidity and a text description of the weather for the city where the patient lives from the Weather Underground website (https://www.wunderground.com/). To take into account that the incubation period of the virus is approximately 14 days, we collected this data for a date 14 days before the patient exhibited relevant symptoms (as recorded in the Novel Corona Virus 2019 dataset).

For a given country, we assume that the researchers' attitude toward COVID-19 will reflect the response capacity of the country, to some extent. For journal publications obtained from the WHO database, we extracted the author's institution with the help of the paper's DOI. We then applied sentiment analysis on each abstract so as to obtain a polarity score for every abstract, and we then calculated an weighted average polarity score for each country. This feature was added to the enhanced dataset. Fig 2 displays the weighted average polarity score inferred for different countries.

Fig 1. Construction of database. Enhancement. The Kaggle COVID-19 database is filtered for patients for which the outcome has been recorded, and then, for these items, the weather is determined using the https://www . wunderground.com website. The WHO COVID-19 literature database is filtered for items for which both a journal name and DOI are provided, and these are post-processed so as to obtain a country-wise research sentiment polarity score. XGBoost is then trained and run on both the initial and the enhanced data and the accuracy of survival prediction is shown to be $85 \%$ and $95 \%$, respectively.

Fig 2. Sentiment polarity of abstract. Average research sentiment polarity score of research, for different countries. Based on a sentiment analysis of abstracts of papers published on COVID-19.

\section{Data processing}

The features obtained from the Kaggle Novel Corona Virus 2019 dataset include both categorical variables and discrete variables. Each item in the dataset contains the variables sex, age, the time interval between the patient's onset date, confirmed infected date and admission date, symptoms description, infection reason and outcome. We will refer to this as the initial dataset.

We added local weather variables (temperature, humidity, climate description) and the weighted polarity score of country's research attitude. We will refer to the result of this as the enhanced dataset.

To prepare for analysis with XGBoost (as discussed below), we tokenized all multi-value text features, such as symptom description or climate description, into 
three-dimensional embedding vectors, used label encoding on categorical variables such as infected reason, as shown in Table 1.

We assigned the constant -999 to all missing values. After filtering for samples that have a valid outcome value, we obtain 183 samples.

Table 1. Example of multi-value text features.

\begin{tabular}{|l|c|}
\hline Features & Example \\
\hline $\begin{array}{l}\text { symptom description } \\
\text { weather description }\end{array}$ & fair, Light Rain Shower, Cloudy \\
\hline
\end{tabular}

\section{Data statistics}

Due to the relatively small size of the two datasets, we split each dataset into a training set and a testing set in the proportion of 8:2. As shown in Table 2, the original dataset is typically imbalanced, and after applying the Synthetic Minority Over-sampling Technique (SMOTE) [9] on the minority group, the ratio of the positive samples and the negative sample is 1:5. Note that, for technical reasons, here positive samples refer to patients that died.

Table 2. Sampling statistics for the enhanced dataset.

\begin{tabular}{|l|c|c|}
\hline Statistic & Enhanced data & After oversampling \\
\hline Positive samples & 15 & 33 \\
Negative samples & 168 & 168 \\
Total & 183 & 201 \\
\hline
\end{tabular}

\section{Methods and experiment}

\section{Sentiment analysis}

A number of papers have studied the forecasting of pandemics using natural language processing on data obtained from various social media $10-12$. Along these lines, we performed sentiment analysis on the abstracts of research papers (associated with COVID-19) using the Python package Textblob (https://github.com/sloria/TextBlob), which operates by analyzing text content and assigns emotional values to word based on matches to a built-in dictionary.

\section{Machine learning algorithm}

Our aim is to predict whether the patient will survive the infection, based on either the initial dataset or the enhanced dataset.

We use the Extreme Gradient Boosting (XGBoost) 13 method to address this. XGBoost is a powerful member of the gradient boosting family, which is designed to perform well on sparse features, and is known to perform well on Kaggle tasks, This approach avoids overfitting using its built-in L1 and L2 regularization on the target function:

$$
O b j=\sum_{i=1}^{n} l\left(y_{i}, \hat{y}_{i}\right)+\sum_{i=1}^{t} \Omega\left(f_{i}\right)
$$

As an additive model, XGBoost consists of $k$ base models, and in most cases we choose the tree model as its base model. Suppose, for the $k$-th of $t$ iterations, that we 
train the tree model $f_{k}(x)$, then

$$
\hat{y}_{i}^{t}=\sum_{k=1}^{t} f_{k}\left(x_{i}\right)=\hat{y}_{i-1}^{t}+f_{t}\left(x_{i}\right)
$$

is the estimate result of the sample $i$ after $t$ times' iteration. During construct of each tree, XGBoost minimizes the objective function with regularization term introduced in Eq (1) in the split phase of each node. In each tree, we calculate the Gain of the feature and choose the tree who has the biggest value as the leaf node to be split:

$$
\text { Gain }=\frac{G_{L}^{2}}{H_{L}+\lambda}+\frac{G_{R}^{2}}{H_{R}+\lambda}-\frac{\left(G_{L}+G_{R}\right)^{2}}{H_{L}+H_{R}+\lambda}-\lambda .
$$

\section{Implementation}

In this study, we ran the XGBoost algorithm on two different datasets, namely the initial dataset and the enhanced dataset, the latter containing additional features representing local weather and research sentiment, as illustrated in Fig 1 .

To obtain the model with the best capacity for prediction, we used a grid search for model tuning. Each subtree in our model is a simple tree whose maximum depth is 3 . The learning rate was 0.01 . During the training step, we randomly sampled the columns of each tree according to a ratio of 0.5 .

\section{Results}

We evaluated the algorithm's performance by calculating each model's classification accuracy. The accuracy of the model created by using the initial dataset (no added features) is $85 \%$, whereas using the enhanced dataset (with added features), the model's accuracy is $95 \%$.

The method we chose to evaluate the importance score of feature is based on counting the number of times that a feature occurred in a tree. The feature importance for both datasets is shown in Fig 3 . For the initial dataset, age plays a more important role than other features. For the model based on the enhanced dataset, weighted average research sentiment polarity score is more important than age, whereas the level of importance of weather is similar to that of age.

Fig 3. Feature scores on initial and enhanced datasets.

(A) Feature scores on the initial dataset.

(B) Feature scores on the enhanced dataset.

\section{Conclusion}

The performance of machine learning methods depends on the amount and quality of available features. For our analysis, we can say that the current publicly available data is poor. First, the data is quite sparse and there are too few features. Here we see that by enhancing the dataset, the accuracy of survival prediction can be increased by $10 \%$.

Our study shows how one might enhance a dataset by adding informative features if they are not available in the original dataset. Here we demonstrated this for country-wise research sentiment and local weather. Local weather conditions has been implicated as an important feature in the existing research.

Our analysis confirms the observation that age is an important factor for survival of COVID-19. However, in the data considered here, the total number of deaths above age 
60 were 8 , and 16 survived or were still alive, while in the age group between 40-60 there were 2 deaths and 36 alive or survived. Hence, linking mortality to a particular age group is not be appropriate based on the current result. While this analysis suggests that elderly have a higher risk of death, which has already been observed [14, 15], saying mortality is associated with old age is probably generally true for any infectious disease. Age is one of the confounding factors that could be responsible for enhanced COVID-19 mortality rate, so more emphasis should be be taken for the elderly care [16, 17].

For the model based on the enhanced dataset, the weighted average of research sentiment, followed by weather and age, appear as the most important features, and account for the increase in the accuracy of the model. This confirms that environmental conditions play a role. Also, it suggests the research sentiment might reflect a countries ability to tackle the disease.

Finally, this analysis suggests that enhancing a dataset, rather than just analyzing the originally given features, might lead to a better prediction of the particular outcome.

\section{Supporting information}

S1 File. Initial COVID-19 dataset 183 cases.

S2 File. Processed WHO publication data.

S3 File. Enhanced COVID-19 dataset.

\section{Funding}

This work was supported by the BMBF-funded de.NBI Cloud within the German Network for Bioinformatics Infrastructure (de.NBI) (031A537B, 031A533A, 031A538A, 031A533B, 031A535A, 031A537C, 031A534A, 031A532B). Also, we acknowledge support by the Open Access Publishing Fund of University of Tübingen.

\section{References}

1. Torales J, O'Higgins M, Castaldelli-Maia JM, Ventriglio A. The outbreak of COVID-19 coronavirus and its impact on global mental health. International Journal of Social Psychiatry. 2020 Mar 31:0020764020915212.

2. Singh J, Singh J. COVID-19 and its impact on society. Electronic Research Journal of Social Sciences and Humanities. 2020 Apr 3;2.

3. Holmes EA, O'Connor RC, Perry VH, Tracey I, Wessely S, Arseneault L, Ballard C, Christensen H, Silver RC, Everall I, Ford T. Multidisciplinary research priorities for the COVID-19 pandemic: a call for action for mental health science. The Lancet Psychiatry. 2020 Apr 15.

4. Narin A, Kaya C, Pamuk Z. Automatic detection of coronavirus disease (covid-19) using x-ray images and deep convolutional neural networks. arXiv preprint arXiv:2003.10849. 2020.

5. Magar R, Yadav P, Farimani AB. Potential neutralizing antibodies discovered for novel corona virus using machine learning. arXiv preprint arXiv:2003.08447. 2020 Mar 18. 
6. Sajadi MM, Habibzadeh P, Vintzileos A, Shokouhi S, Miralles-Wilhelm F, Amoroso A. Temperature and latitude analysis to predict potential spread and seasonality for COVID-19. Available at SSRN 3550308. 2020 Mar 5.

7. Lin K, Fong DY, Zhu B, Karlberg J. Environmental factors on the SARS epidemic: air temperature, passage of time and multiplicative effect of hospital infection. Epidemiology \& Infection. 2006 Apr;134(2):223-30.

8. Triplett M. Evidence that higher temperatures are associated with lower incidence of COVID-19 in pandemic state, cumulative cases reported up to March 27, 2020. medRxiv. 2020.

9. Chawla NV, Bowyer KW, Hall LO, Kegelmeyer WP. SMOTE: synthetic minority over-sampling technique. Journal of artificial intelligence research. 2002 Jun $1 ; 16: 321-57$.

10. Alessa A, Faezipour M. A review of influenza detection and prediction through social networking sites. Theoretical Biology and Medical Modelling. 2018 Dec $1 ; 15(1): 2$.

11. Lee K, Agrawal A, Choudhary A. Forecasting influenza levels using real-time social media streams. In2017 IEEE International Conference on Healthcare Informatics (ICHI) 2017 Aug 23 (pp. 409-414). IEEE.

12. Wang Y, Xu K, Kang Y, Wang H, Wang F, Avram A. Regional influenza prediction with sampling twitter data and PDE model. International journal of environmental research and public health. 2020 Jan;17(3):678.

13. Chen Tianqi, and Carlos Guestrin. Xgboost: A scalable tree boosting system. Proceedings of the 22nd acm sigkdd international conference on knowledge discovery and data mining, 2016.

14. Verity R, Okell LC, Dorigatti I, Winskill P, Whittaker C, Imai N, Cuomo-Dannenburg G, Thompson H, Walker PG, Fu H, Dighe A. Estimates of the severity of coronavirus disease 2019: a model-based analysis. The Lancet infectious diseases. 2020 Mar 30.

15. Glynn JR. Protecting workers aged 60-69 years from COVID-19. The Lancet Infectious Diseases. 2020 Apr 16.

16. Wang H, Li T, Barbarino P, Gauthier S, Brodaty H, Molinuevo JL, Xie H, Sun Y, Yu E, Tang Y, Weidner W. Dementia care during COVID-19. The Lancet. 2020 Apr 11;395(10231):1190-1.

17. Armitage R, Nellums LB. COVID-19 and the consequences of isolating the elderly. The Lancet Public Health. 2020 May 1;5(5):e256. 


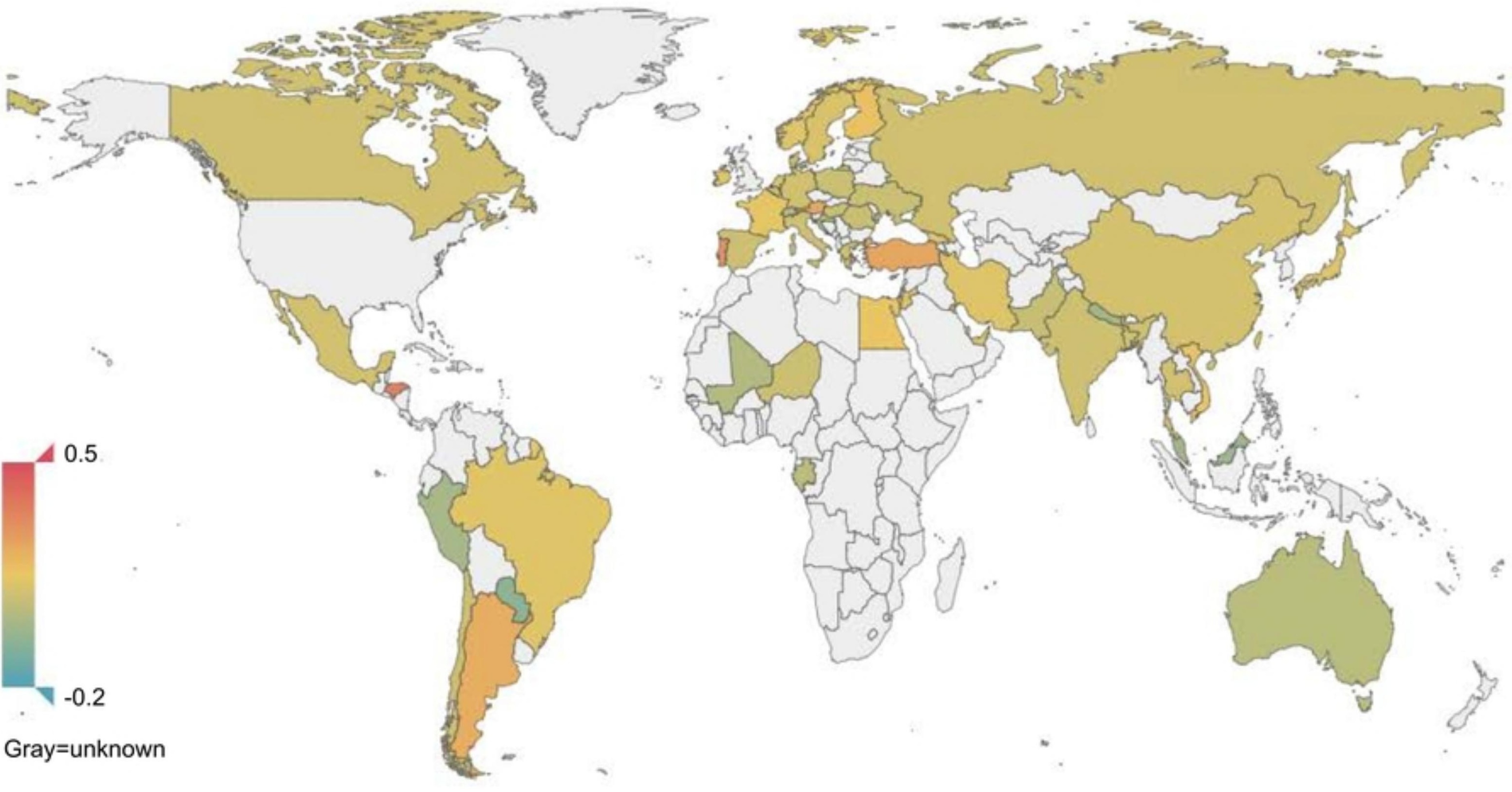




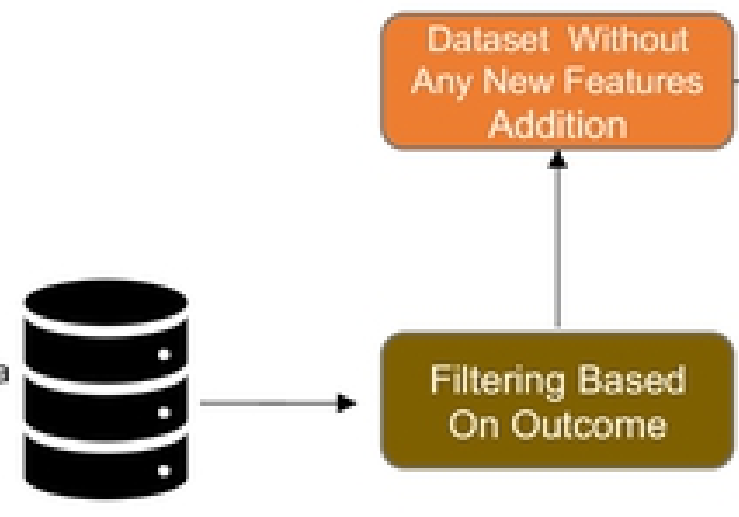

Filtering Based

$\underset{\substack{\text { Country } \\ \text { input }}}{\substack{\text { Names }\\}}$

Weather

Underground

Website

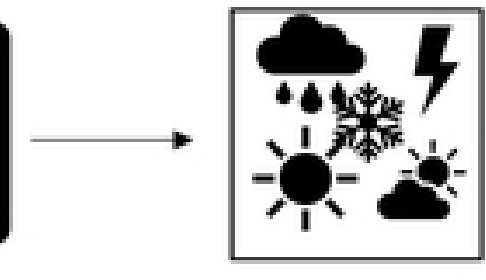

Weather Condition For Past 14 Days From Date of

$$
\text { Onset }
$$

ML

Processing XGBosst
WHO COVID-19

Database
Publication datasheet

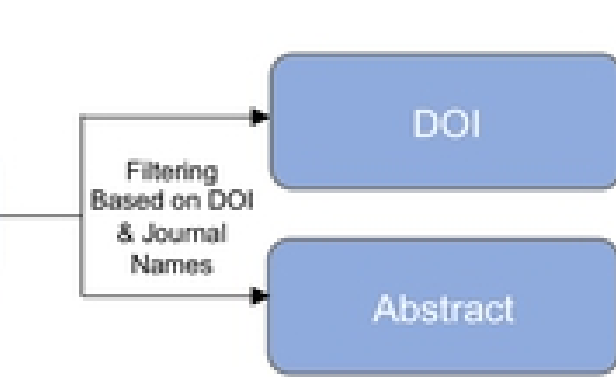

Data
Crawing
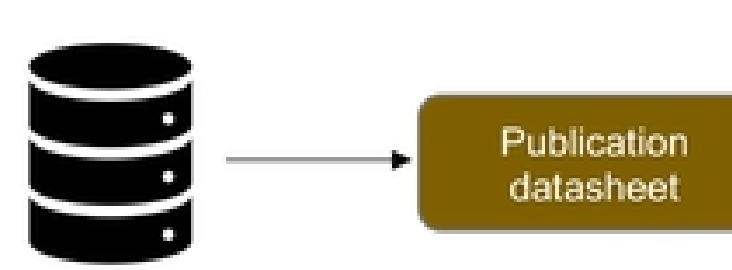
Feature Importance (Model Accuracy 95\%)

comfirm onset delta confirm_admission_delta

hum 0

hum 1

hum $\overline{1} 0$

hum-11

hum 12

hum 13

hum 14 .

hum 2

hum 3 .

hum 4

hum 6 .

hum 7

hum 8 .

hum 9

infected_reasōn

sex

symptoms_emb 0 symptoms emb 1 .

symptoms_emb_2

temp 0 -

temp 1

temp $\overline{1} 0$.

temp_11.

temp_12

temp_13

temp 14

temp_2.

temp 3 .

temp 4

temp 6

temp 7

temp_8

temp 5

weather_emb 0 .

weather_emb_1.

weather_emb-2. weighted_āveraḡe

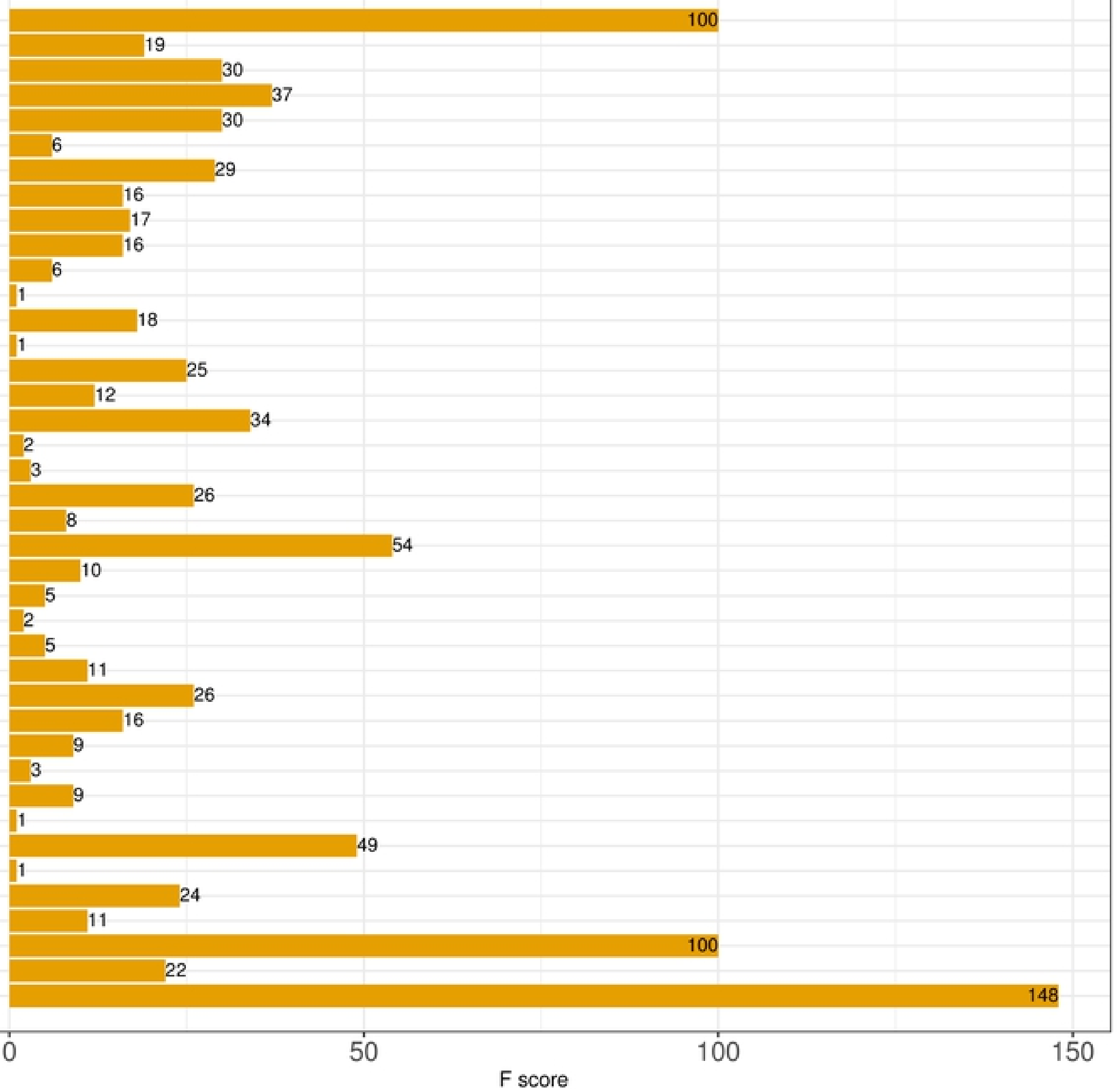




\section{Feature Importance (Model Accuracy $85 \%$ )}

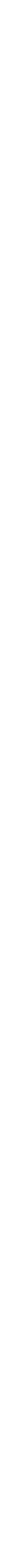

